\title{
The Response of the Tropospheric Circulation to Water Vapor-Like Forcings in the Stratosphere
}

\author{
NEIL F. TANDON \\ Department of Applied Physics and Applied Mathematics, Columbia University, New York, New York \\ LORENZO M. POLVANI \\ Department of Applied Physics and Applied Mathematics, and Department of Earth and Environmental Sciences, \\ Columbia University, New York, New York \\ SEAN M. DAVIS \\ Chemical Sciences Division, NOAA/Earth System Research Laboratory, and Cooperative Institute for Research \\ in Environmental Science, University of Colorado, Boulder, Colorado
}

(Manuscript received 2 February 2011, in final form 11 April 2011)

\begin{abstract}
An idealized, dry general circulation model is used to examine the response of the tropospheric circulation to thermal forcings that mimic changes in stratospheric water vapor (SWV). It is found that SWV-like cooling in the stratosphere produces a poleward-shifted, strengthened jet and an expanded, weakened Hadley cell. This response is shown to be almost entirely driven by cooling located in the extratropical lower stratosphere; when cooling is limited to the tropical stratosphere, it generates a much weaker and qualitatively opposite response. It is demonstrated that these circulation changes arise independently of any changes in tropopause height, are insensitive to the detailed structure of the forcing function, and are robust to model resolution. The responses are quantitatively of the same order as those due to well-mixed greenhouse gases, suggesting a potentially significant contribution of SWV to past and future changes in the tropospheric circulation.
\end{abstract}

\section{Introduction}

Water vapor is the dominant greenhouse gas of Earth's atmosphere, but important questions remain about its future changes and their impact on climate. This is particularly true of stratospheric water vapor (SWV), whose abundance depends on numerous factors-most importantly, methane oxidation and tropical cold-point temperatures (e.g., Fueglistaler et al. 2009)—for which predictions are highly uncertain (e.g., Solomon et al. 2007, chapter 10). Data over select regions have shown a $\sim 0.5$ ppmv decade ${ }^{-1}$ increase in stratospheric water vapor for much of the twentieth century (Rosenlof et al. 2001; Hurst et al. 2011). After a decrease of $\sim 0.4$ ppmv between 2001 and 2005 (Randel et al. 2006;

Corresponding author address: Neil F. Tandon, Columbia University, 500 W. 120 St., Room 200 Mudd, New York, NY 10027. E-mail: nft2104@columbia.edu
Solomon et al. 2010), SWV levels have increased $\sim 0.5$ ppmv since 2006 (Hurst et al. 2011).

As for the effects of SWV changes, earlier studies have focused almost exclusively on the radiatively forced temperature response. Using fixed dynamical heating (FDH) and energy balance models, Forster and Shine (2002) have shown that an increase in SWV causes cooling in the stratosphere (enhanced in the extratropical lower stratosphere) and warming at the surface. This agrees with the FDH calculations of Maycock et al. (2011) and R. Portmann (2010, personal communication), and with the general circulation model (GCM) simulations of Oinas et al. (2001) and Smith et al. (2001). That said, the circulation response to SWV change has received very little attention. One study (Joshi et al. 2006), using a comprehensive GCM, has shown that SWV increase is a significant contributor to recent strengthening of the North Atlantic Oscillation.

This leaves open several important questions which we consider in this paper: First, how does SWV change 
(a) Our control integration

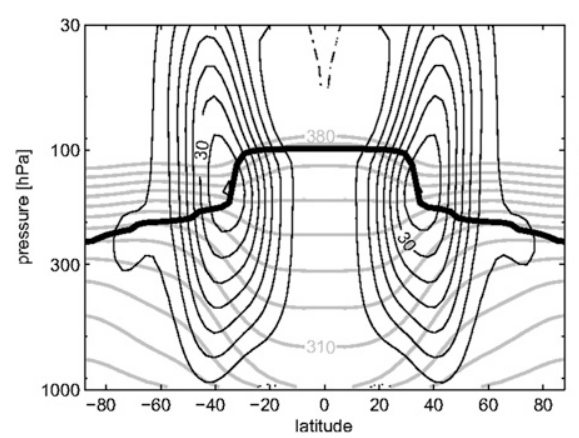

(b) Held and Suarez (1994)

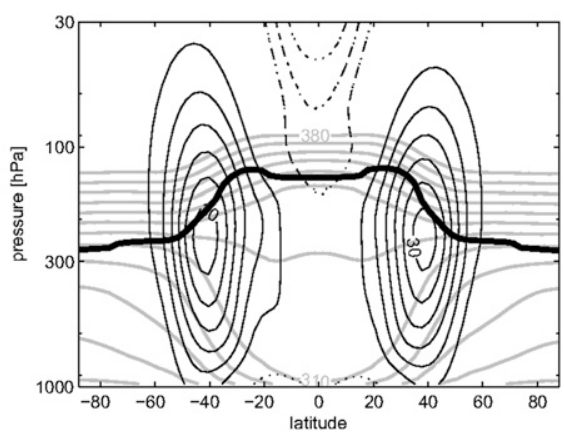

FIG. 1. Zonal mean climatology of (a) our control integration using S04/SW06 parameterizations (see appendix for details) and (b) the Held and Suarez (1994) configuration, shown for comparison. Black contours are zonal wind, with contour interval $5 \mathrm{~m} \mathrm{~s}^{-1}$, negative contours dotted, and zero contour omitted. The thick black contour is the thermally-defined tropopause (World Meteorological Organization 1957). Gray contours are the potential temperature, with contour interval $10 \mathrm{~K}$, and contours above $380 \mathrm{~K}$ omitted; the $380-\mathrm{K}$ isentrope marks the approximate height of our climatological tropical tropopause.

impact the global circulation-both tropical and extratropical? Second, is the tropospheric circulation response driven preferentially by specific regions of stratospheric cooling? Third, how does the circulation response vary with the amount of SWV change? To address these questions, we employ an idealized, dry GCM in which we impose thermal forcings that mimic the effects of SWV. Using such a model affords greater control over the placement and magnitude of the thermal forcing. Thus we develop a clearer picture of not only the global circulation response to SWV but also the precise contributors to that response.

\section{Method}

In this study, we use a model consisting of only dry dynamics and highly idealized parameterizations borrowed from Schneider (2004, hereafter S04) and Schneider and Walker (2006, hereafter SW06). In contrast to the more popular Held and Suarez (1994) configuration (e.g., Polvani and Kushner 2002; Haigh et al. 2005; Lorenz and DeWeaver 2007; Butler et al. 2010), our choices produce a tropical climatology that is closer to observations: the tropical tropopause reaches a potential temperature of $370-380 \mathrm{~K}$, and static stability reflects a moist adiabatic lapse rate (Fig. 1a), compared to the 340-350-K tropopause and dry adiabatic lapse rate of Held and Suarez (1994) (Fig. 1b). For complete reproducibility, all model details are provided in the appendix. Suffice it to say, our model produces a hemispherically symmetric mean circulation, resembling equinoctial conditions.

To study SWV changes, we impose a number of thermal forcings, consisting of perturbations of the model's radiative equilibrium temperature. The amplitude of each perturbation is controlled primarily by a parameter $\delta T$, and additional parameters control its latitudinal and vertical structure. In Fig. 2, we provide visual examples, with complete details available in the appendix. The "LS" (i.e., lower stratosphere) forcing (e.g., Fig. 2a) mimics the temperature response due to a uniform increase in SWV, with strong cooling in the extratropical lower stratosphere and weaker cooling in the tropics and higher altitudes (Forster and Shine 2002; Maycock et al. 2011). As a point of reference, the LS-NEG5 case $(\delta T=$ $-5 \mathrm{~K}$ ) corresponds roughly to a 5 ppmv increase in SWV.

With the "ELS" forcing (e.g., Fig. 2b), we isolate the extratropical portion of the LS perturbation, and with the "TLS" forcing (e.g., Fig. 2c) we isolate the tropical portion. As an additional test, we apply the "LSdp" forcing (e.g., Fig. 2d), in which the LS perturbation is shifted in altitude. When considering specific perturbation amplitudes, we append the above shorthand appropriately; for example, "LS-POS5" indicates a LS forcing integration with nominal amplitude $\delta T=+5 \mathrm{~K}$.

For each of the above forcing functions-and for each choice of $\delta T$-we start our model from rest and integrate for 10000 days, which is sufficient to obtain a statistically stationary response. To compute all climatological fields, we discard the first 300 days as spinup and time average the rest. To obtain the "response" of the model, we subtract the climatology of the control integration, in which $\delta T=0$ (shown in Fig. 1a). Since there is no topography in this model, and all forcings are hemispherically symmetric, all responses should be hemispherically symmetric. Any asymmetry that remains is due to sampling error. Unless otherwise stated, all integrations are 
(a) LS-NEG5

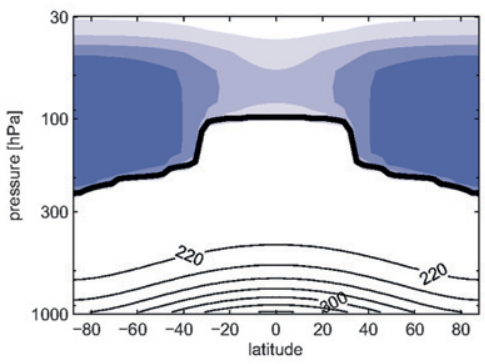

(c) TLS-NEG10

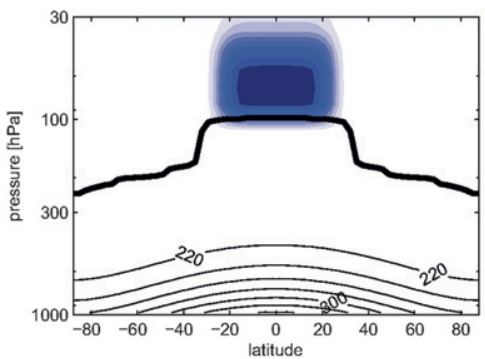

(b) ELS-NEG5

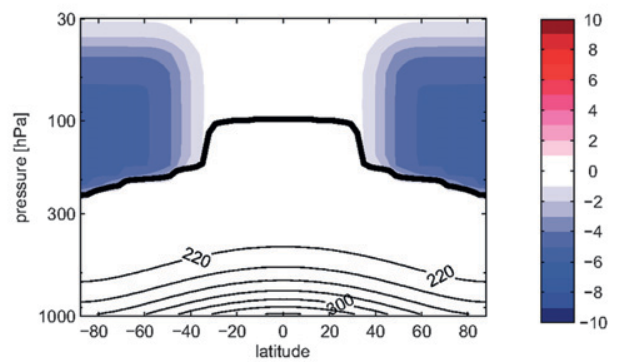

(d) LSdp-NEG5

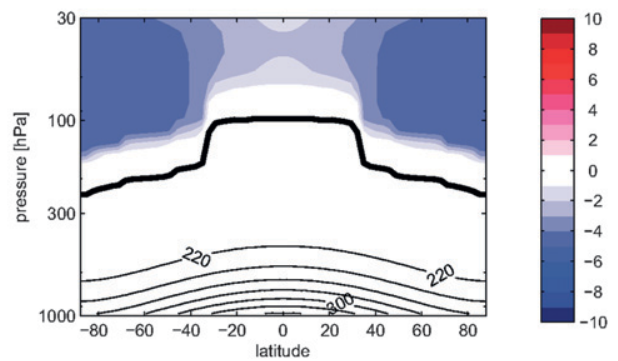

FIG. 2. Color shading shows the different thermal forcings used in this study, described in section 2: (a) LS forcing, with $\delta T=-5 \mathrm{~K}$, constructed to mimic Forster and Shine (2002); (b) ELS forcing, with $\delta T=-5 \mathrm{~K}$; (c) TLS forcing, with $\delta T=-10 \mathrm{~K}$; and (d) as in (a), but shifted upward. The shading interval is $1 \mathrm{~K}$. Thin black contours are the radiative equilibrium temperature of the control integration (i.e., $\delta T=0$ ), with contour interval $20 \mathrm{~K}$. Thick black contour is the thermal tropopause of the control integration.

performed at spectral resolution T42 with 40 vertical levels. (See appendix for details.)

\section{Results}

We first consider the effect of a thermal perturbation that mimics a 5 ppmv increase in $\mathrm{SWV}$, corresponding to our LS-NEG5 forcing. The response is shown in Fig. 3, left column. Because the stratosphere is close to radiative equilibrium, the temperature response (Fig. 3a) shows a strong resemblance to the thermal forcing (Fig. 2a). The cooling in the stratosphere produces a $\sim 30 \mathrm{hPa}$ rise in polar tropopause height (thick dotted contour), with gradually vanishing tropopause changes toward lower latitudes.

Figure $3 \mathrm{~b}$ shows the zonal wind response to the LSNEG5 forcing. There is deceleration of $\sim 1.5 \mathrm{~m} \mathrm{~s}^{-1}$ on the equatorward flank of the jet, accompanied by $\sim 1.5 \mathrm{~m} \mathrm{~s}^{-1}$ acceleration on its poleward flank, indicating a poleward shift of the jet. Figure $3 \mathrm{c}$ shows the changes in the meridional mass streamfunction $\Psi$ (see Peixoto and Oort 1992, section 7.4.3 for the definition). In the northern hemisphere, there is a slight decrease in $\Psi$ within the Hadley cell, a more substantial increase in $\Psi$ at the Hadley cell edge, and a substantial decrease in $\Psi$ near the poleward edge of the Ferrel cell. These features indicate a weakening and widening of the Hadley cell and a poleward migration of the Ferrel cell. The same interpretation applies in the southern hemisphere but with the sign of $\Psi$ reversed.

A natural question arises next: which latitudes of SWV change contribute most to this response? To answer this, we show the ELS-NEG5 integration, in which cooling is confined to the extratropical lower stratosphere (Fig. 3d). In this case, the tropospheric response is nearly indistinguishable from LS-NEG5, showing a poleward shift of the jets (Fig. 3e), expansion and weakening of the Hadley cells, and a poleward shift of the Ferrel cells (Fig. 3f). This suggests that most of the tropospheric response in LS-NEG5 is attributable to cooling in the extratropical lower stratosphere.

To complement ELS-NEG5, we also present the TLSNEG10 integration, in which cooling is confined to the tropical lower stratosphere (Fig. 3g). To elucidate the tropospheric response, which is weaker than with extratropical forcing, we here increase the perturbation amplitude to $\delta T=-10 \mathrm{~K}$. Notice that with tropical forcing, the response is qualitatively opposite to that of LS-NEG5 and ELS-NEG5, with a slight equatorward shift of the jets (Fig. 3h), contraction of the Hadley cells, and an equatorward shift of the Ferrel cells (Fig. 3i). For lower amplitudes of tropical cooling (not shown), 
LS-NEG5

(a) $\Delta T[\mathrm{~K}]$

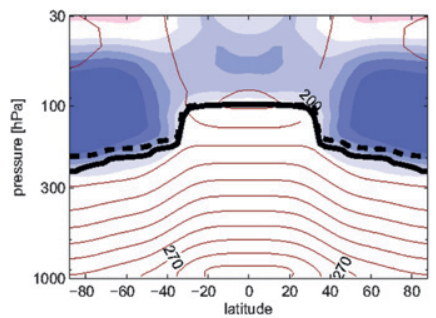

(b) $\Delta u\left[\mathrm{~m} \mathrm{~s}^{-1}\right]$

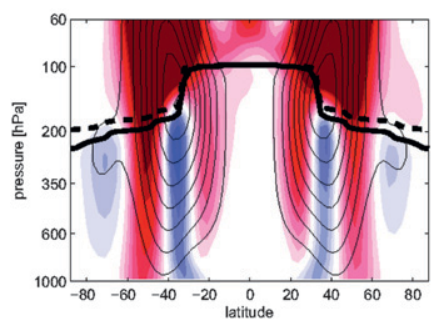

(c) $\Delta \Psi\left[10^{9} \mathrm{~kg} \mathrm{~s}^{-1}\right]$

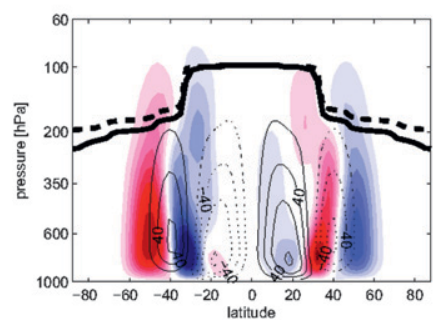

ELS-NEG5

(d) $\Delta T[\mathrm{~K}]$

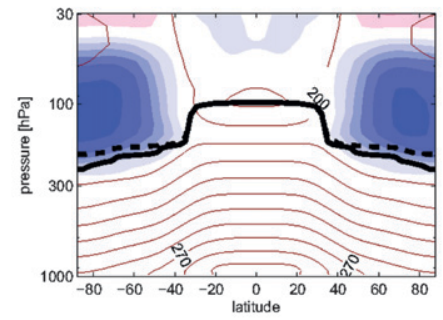

(e) $\Delta u\left[\mathrm{~m} \mathrm{~s}^{-1}\right]$

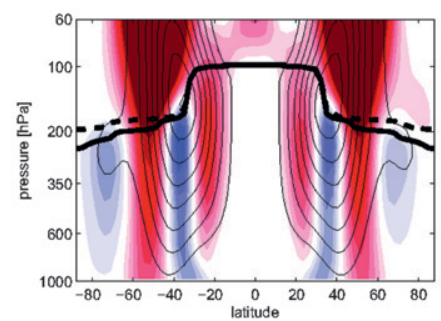

(f) $\Delta \Psi\left[10^{9} \mathrm{~kg} \mathrm{~s}^{-1}\right]$

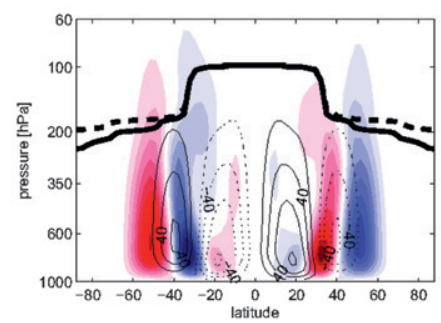

TLS-NEG10

(g) $\Delta T[\mathrm{~K}]$

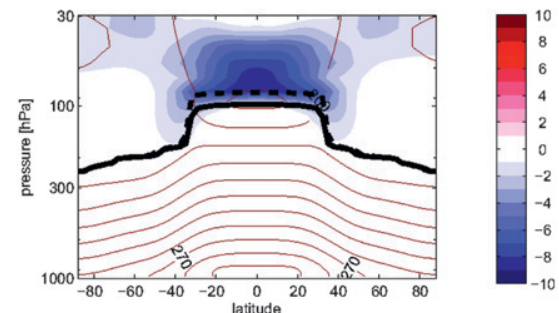

(h) $\Delta u\left[\mathrm{~m} \mathrm{~s}^{-1}\right]$

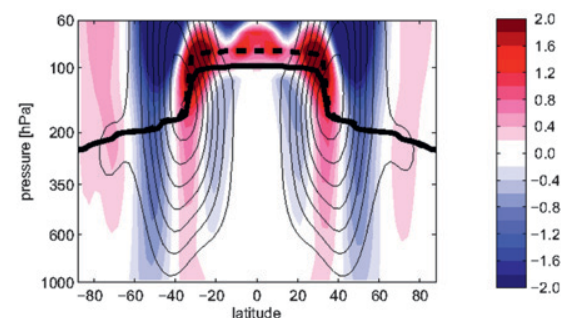

(i) $\Delta \Psi\left[10^{9} \mathrm{~kg} \mathrm{~s}^{-1}\right]$

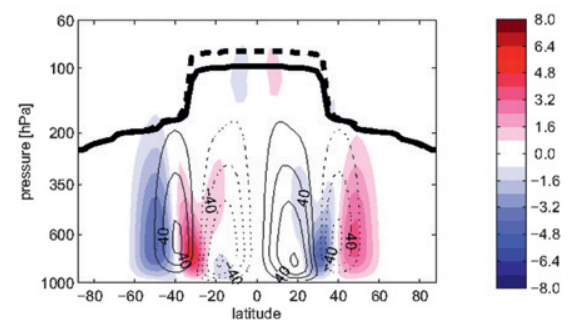

FIG. 3. Color shading is the response to the specific forcings, as indicated at the top of each column. Thin black contours are the climatology of the control integration. Solid, thick black contour is the thermal tropopause of the control integration. The dotted, thick, black contour is the tropopause of the perturbed integration. Contour intervals are (top) $10 \mathrm{~K}$ for temperature; (middle) $5 \mathrm{~m} \mathrm{~s}^{-1}$ for zonal wind with contours below $5 \mathrm{~m} \mathrm{~s}^{-1}$ omitted; and (bottom) $20 \times 10^{9} \mathrm{~kg} \mathrm{~s}^{-1}$ for the meridional mass streamfunction with negative contours dashed and zero contour omitted.

qualitatively similar features result, but the response is very weak and much longer integrations are required to reach equilibrium. These findings confirm that, in LSNEG5 (Figs. 3a-c), cooling in the tropical stratosphere contributes negligibly to the tropospheric response.

We have extensively explored the space of forcing functions and amplitudes, and Fig. 4 summarizes the results. Each panel shows several curves, each one representing a set of integrations with the same thermal forcing structure but different forcing amplitudes. For stratospheric cooling $(\delta T<0)$, both the LS (red circles) and ELS (blue circles) forcings produce poleward-shifted jets (Fig. 4a) and expanded Hadley cells (Fig. 4b). Because of the hemispheric symmetry of our model, expansion of the Hadley cells indicates a poleward shift of the Hadley cell edges, which also indicates poleward migration of the Ferrel cells. These results are also apparent from Fig. 3, discussed above. For $\delta T<0$, the LS and ELS forcings also produce an increase in jet speed (Fig. 4c) and a weakening of the Hadley circulation (Fig. 4d). All these results, one should note, are robust to changes in the model's vertical resolution. Whether it is doubled to 80 levels (red squares) or halved to 20 levels (red crosses), the results are essentially identical to the standard 40 -level case, over the full range of $\delta T$.

The TLS integrations, with forcing confined to the tropics, are indicated by the green circles in Fig. 4. TLS shows a $\delta T$ dependence opposite to that of LS and ELS, as already illustrated in Fig. 3, and the response is comparatively small over the full range of $\delta T$. This further confirms that the tropical stratosphere contributes very little to our model's response. This does not mean, however, that the LS results are simply the linear 
(a) Jet Latitude

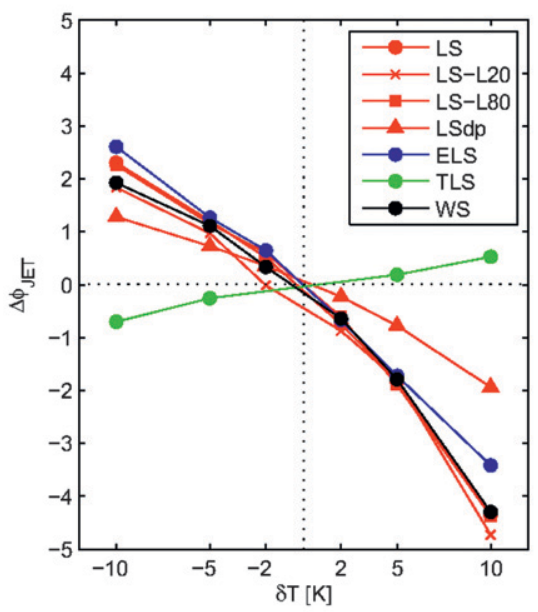

(c) Jet Speed

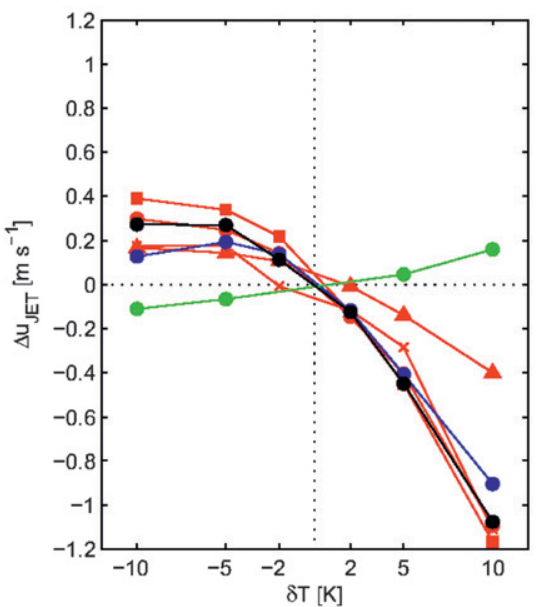

(b) Hadley Cell Width

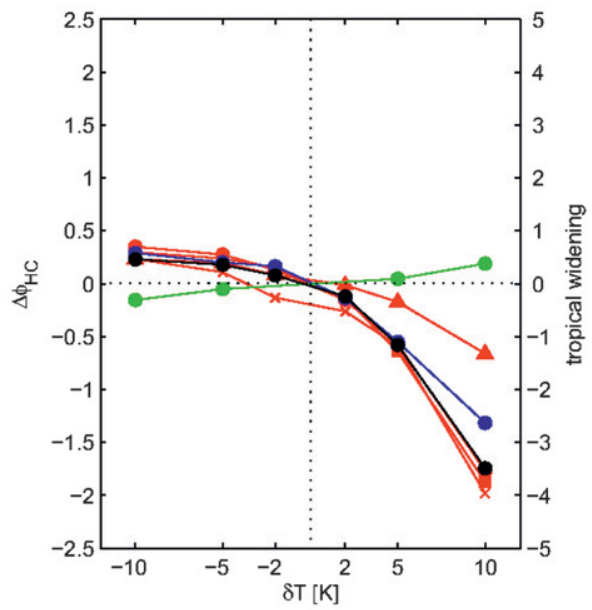

(d) Hadley Cell Strength

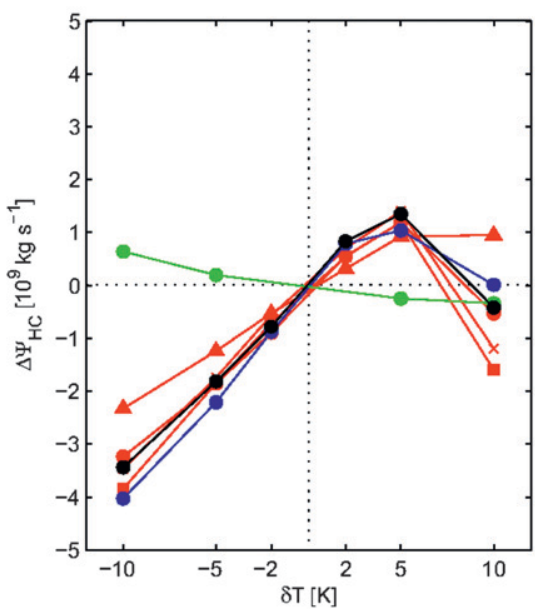

FIG. 4. Changes in circulation metrics as functions of nominal perturbation amplitude, $\delta T$. (a) The change in jet latitude, defined as the latitude of maximum zonal wind at the lowest model level. This metric isolates changes in the extratropical eddy-driven component of the jet. (b) The change in Hadley cell width, defined as $\phi_{\Psi 02}-\phi_{\Psi 01}$, where $\phi_{\Psi 0 \mathrm{i}}$ is the $i$ th zero crossing of $\Psi$ at $500 \mathrm{hPa}$, starting nearest the equator. The right-hand vertical axis multiplies the change in Hadley cell width by two to measure the total tropical widening (cf. Seidel et al. 2008; Johanson and $\mathrm{Fu}$ 2009). (c) The change in jet speed, defined as the maximum in zonal wind at the lowest model level. (d) The change in Hadley cell strength, defined as the maximum of $|\Psi|$ between $\phi_{\Psi 01}$ and $\phi_{\Psi 02}$. Northern and Southern Hemisphere values are averaged together.

composite of ELS and TLS. For example, the shift of the jet in the LS-POS10 case $\left(-4.34 \pm 0.04^{\circ}\right)$ is actually larger in magnitude than for ELS-POS10 $\left(-3.42 \pm 0.04^{\circ}\right)$, while linearity would suggest a smaller shift.

The results of Fig. 4 are nonlinear in another sense: the response to stratospheric warming $(\delta T>0)$ is not simply the reverse of the one associated with stratospheric cooling $(\delta T<0)$. This nonlinearity is most extreme in the cases of jet speed (Fig. 4c) and Hadley cell strength (Fig. 4d), which even show some nonmonotonicity.

\section{Discussion}

We first offer a few points of comparison with earlier work. Joshi et al. (2006) simulate a SWV change corresponding to roughly a $-1-\mathrm{K}$ perturbation of the northern extratropical stratosphere. The magnitude of their zonal 
wind response is somewhat larger than what we obtain in our LS-NEG2 integration (not shown). Note, however, that Joshi et al. (2006) consider winter averages rather than the equinoxes and that their model includes topography and a polar vortex, which may explain the quantitative difference. The differences are more drastic when we compare with earlier idealized studies. For example, the zonal wind responses in Haigh et al. (2005) and Lorenz and DeWeaver (2007) are greater than ours by factors of $2-4$, when considering comparable forcing functions. The reasons for this model-dependence require further study. Nonetheless, our results are in qualitative agreement with earlier work.

Second, as noted earlier, the thermal forcings we have imposed produce substantial changes in tropopause height. One might argue that the tropospheric circulation response we have shown is a simple consequence of tropopause height change, a finding that is welldocumented in the literature (e.g., Williams 2006; Lorenz and DeWeaver 2007). To show that tropopause change is not here the primary cause of the tropospheric circulation response, we present an additional series of integrations, labeled LSdp, in which the stratospheric forcing (e.g., Fig. 2d) is identical to the LS forcing (e.g., Fig. 2a) but shifted higher so as not to affect tropopause height. The response of LSdp-NEG5, shown in Fig. 5, is qualitatively almost identical to the one in Figs. 3a-c, with a slightly smaller amplitude, while tropopause height is basically unchanged. We have verified that this behavior holds over the entire range of forcing amplitudes, as indicated by the red triangles in all panels of Fig. 4 .

We also note that there are slight structural differences between our analytically constructed thermal forcing and the thermal response to SWV increase shown in Forster and Shine (2002). For instance, SWV-caused cooling decreases more gradually with height than in our LS forcing. One might wonder how sensitive our results are to the details of our forcing function. To address this question, we have conducted a series of integrations, labeled "WS" (i.e., whole stratosphere), in which we impose uniform cooling of the entire stratosphere; that is, we cool every point above the tropopause by the same amount $\delta T$, irrespective of height and latitude. The results (Fig. 4, black circles) show that, over the full range of $\delta T$, there is little difference between this simplest WS forcing and the original LS forcing (red circles) that was constructed to mimic Forster and Shine (2002). This further establishes the robustness of our results.

Whether one considers our $\delta T=-5 \mathrm{~K}$ integrations, or more conservatively the $\delta T=-2 \mathrm{~K}$ results (which scale almost linearly), one conclusion remains the same: changes in SWV-in particular extratropical SWVgenerate circulation responses that are of the same order
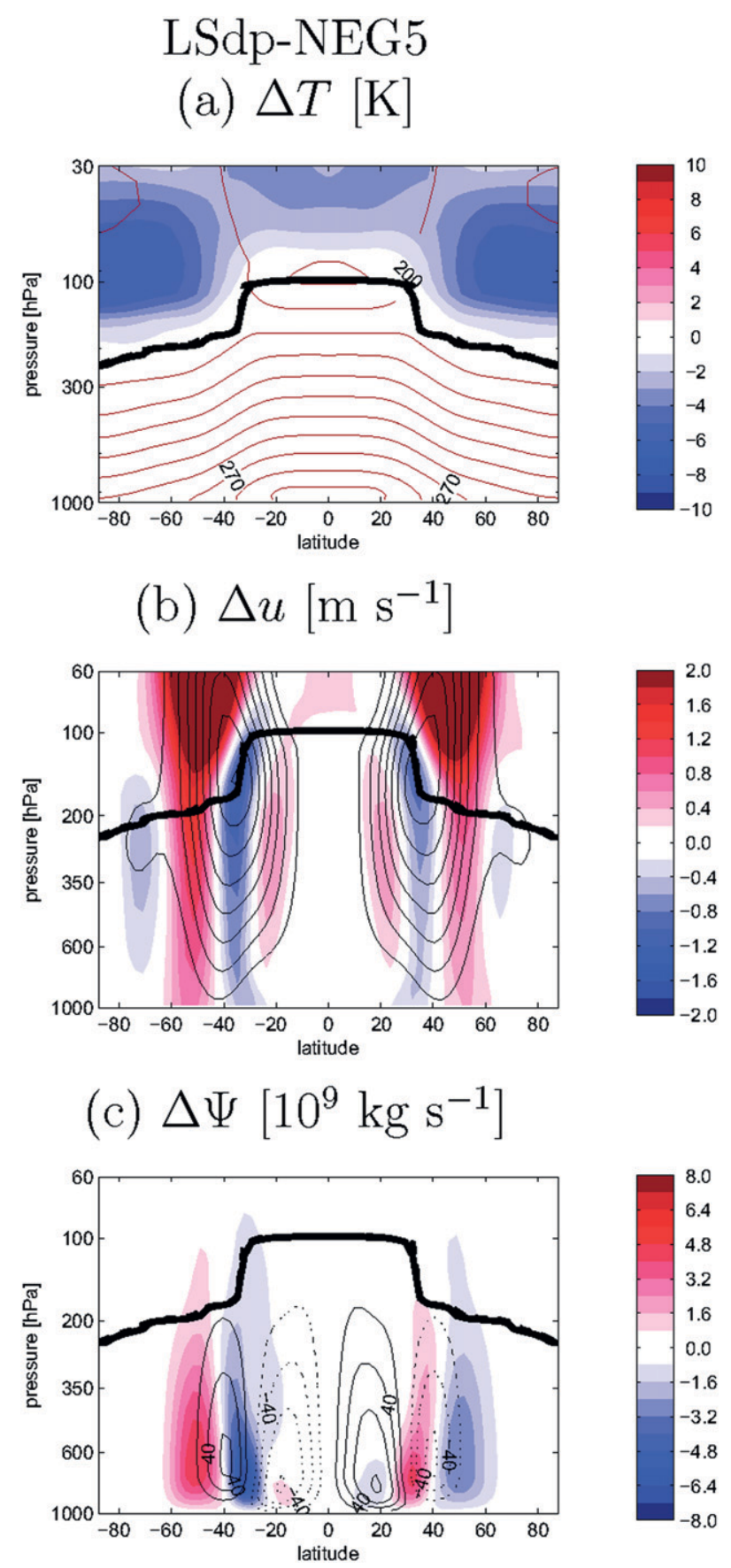

FIG. 5. As in Fig. 3, but for the LSdp-NEG5 integration.

as the modeled response to increased well-mixed greenhouse gases. This is clear, for instance, when comparing the tropical widening in Fig. $4 \mathrm{~b}$ with Fig. 2 of Johanson and $\mathrm{Fu}$ (2009). Indeed, this response is small when compared to observed Hadley cell expansion, but models in general have failed to reproduce this recent trend (Seidel et al. 2008; Johanson and Fu 2009), so the dominant contributors to tropical widening remain unclear. As for 
the extratropical circulation, our results show responses that are significant in the context of both past changes and future projections; compare the zonal wind responses of Figs. $3 \mathrm{~b}$ and $3 \mathrm{e}$ with Figs. 3 and 6 of Lorenz and DeWeaver (2007). Thus SWV is clearly a key component of extratropical circulation change, and it may prove significant to tropical circulation change as well.

Acknowledgments. We thank Karen Rosenlof and Shuguang Wang for helpful discussions and three anonymous reviewers for constructive feedback. NFT and LMP are supported by a grant from the U.S. National Science Foundation. SMD is supported by NASA Aura Grant 07-ASTR07-0004 and the NOAA ACC program.

\section{APPENDIX}

\section{Model Description}

We use the spectral dynamical core of the Geophysical Fluid Dynamics Laboratory (GFDL) Flexible
Modeling System (FMS), which employs the spectral transform method (Hoskins and Simmons 1975) in the horizontal and the finite-difference method (Simmons and Burridge 1981) in the vertical. The horizontal truncation is T42. The vertical level interfaces, in sigma coordinates, are $\sigma_{i}=(i / L)^{3}, i=0,1,2, \ldots, L$, where $L$ is an integer. For all integrations, $L=40$ unless otherwise stated.

To mimic convective and radiative processes, we employ two linear relaxation terms in the temperature equation,

$$
\frac{\partial T}{\partial t}=\cdots-\frac{T-T_{C}}{\tau_{C}}-\frac{T-\left(T_{R}+T^{*}\right)}{\tau_{R}},
$$

where $T_{C}$ and $\tau_{C}$ are the convective equilibrium temperature and time scale, respectively; $T_{R}$ and $\tau_{R}$ are the radiative equilibrium temperature and time scale, respectively; and $T^{*}$ is our external thermal forcing.

As in SW06, $T_{C}$ is given by

$$
T_{C}(\lambda, \phi, p, t)= \begin{cases}T_{m}(\lambda, \phi, p, t)-E_{C}(\lambda, \phi, t) & p_{\mathrm{LNB}}(\lambda, \phi, t) \leq p \leq p_{0} \\ T(\lambda, \phi, p, t) & p<p_{\mathrm{LNB}}(\lambda, \phi, t),\end{cases}
$$

where

$$
E_{C}(\lambda, \phi, t)=\frac{1}{p_{\mathrm{LNB}}(\lambda, \phi, t)-p_{0}} \int_{p_{0}}^{p_{\mathrm{LNB}}(\lambda, \phi, t)}\left[T_{m}\left(\lambda, \phi, p^{\prime}, t\right)-T\left(\lambda, \phi, p^{\prime}, t\right)\right] d p^{\prime}
$$

ensures conservation of enthalpy in (A2); $T_{m}$ is the moist adiabat,

$$
T_{m}(\lambda, \phi, p, t)=T_{s}(\lambda, \phi, t)\left(\frac{p}{p_{0}}\right)^{R \Gamma_{m} / g}
$$

$T_{S}$ is the surface temperature at longitude-latitude-time $(\lambda, \phi, t) ; \Gamma_{m}=7 \mathrm{~K} \mathrm{~km}^{-1} ; p_{0}=1000 \mathrm{hPa}$; and $p_{\mathrm{LNB}}$ is the level of neutral buoyancy for ascent from the surface along $T_{m}$ (e.g., Bohren and Albrecht 1998, section 6.7). Equation (A2) is applicable only when $E_{C} \geq 0$. If $E_{C}<0$, then convection is inhibited; that is, $T_{C}=T$ in the entire column. The time scale $\tau_{C}$ is set to $4 \mathrm{~h}$.

As in $\mathrm{S} 04, T_{R}$ is given by

$$
T_{R}(\phi, p)=T_{\text {strat }}\left[1+d_{0}(\phi)\left(\frac{p}{p_{0}}\right)^{3.5}\right]^{1 / 4}
$$

where

$$
d_{0}(\phi)=\left(\frac{T_{0}+\Delta_{h} \cos ^{2} \phi}{T_{\text {strat }}}\right)^{4}-1
$$

$T_{\text {strat }}=200 \mathrm{~K}, T_{0}=260 \mathrm{~K}$, and $\Delta_{h}=90 \mathrm{~K}$ (see contours in Fig. 2). The time scale $\tau_{R}$ has a vertical and latitudinal dependence given by

$\tau_{R}^{-1}(\phi, \sigma)=\tau_{a}^{-1}+\left(\tau_{s}^{-1}-\tau_{a}^{-1}\right) \max \left(0, \frac{\sigma-\sigma_{b}}{1-\sigma_{b}}\right) \cos ^{8} \phi$,

where $\sigma=p / p_{0}, \tau_{a}=50 \mathrm{~d}, \tau_{s}=7 \mathrm{~d}$, and $\sigma_{b}=0.7$ (S04). We have also tested a more realistic configuration in which $T_{R}$ increases and $\tau_{R}$ decreases in the middle-upper stratosphere. While this slightly affects the magnitudes of our results, there is no qualitative change.

The thermal forcing $T^{*}$ is the only term that we vary among integrations, and it takes the form

$$
T^{*}(\phi, p)=\delta T\left[\frac{1}{1+e^{-s_{p}\left(p-p_{1}\right)}}-\frac{1}{1+e^{-s_{p}\left(p-p_{2}\right)}}\right] w(\phi),
$$

where $\delta T$ controls the forcing amplitude and $s_{p}=$ $0.2 \mathrm{hPa}^{-1}$. The parameters $p_{1}, p_{2}$, and $w(\phi)$ control the 
TABLE A1. Thermal forcing parameters used in Eq. (A8): $p_{1}$ and $p_{2}$ are the approximate lower and upper boundaries, respectively, of the forcing region, and $p_{t}(\phi)$ is the calculated, zonally averaged thermal tropopause of the control integration. In the last column, $\phi$ is the latitude measured in degrees. Additional labels are used in the text to indicate the nominal perturbation amplitude, $\delta T$ (e.g., LS-NEG5).

\begin{tabular}{lllll}
\hline \hline Forcing & \multicolumn{1}{c}{ Description } & $p_{1}(\mathrm{hPa})$ & $p_{2}(\mathrm{hPa})$ & $w(\phi)$ \\
\hline LS & lower stratosphere (Fig. 2a) & $p_{t}(\phi)$ & 40 & $1-0.4 e^{-(\phi / 40)^{2}}$ \\
ELS & extratropical lower stratosphere (Fig. 2b) & $p_{t}(\phi)$ & 40 & $1-e^{-(\phi / 45)^{6}}$ \\
TLS & tropical lower stratosphere (Fig. 2c) & 98.5 & 40 & $e^{-(\phi / 25)^{6}}$ \\
LSdp & LS, shifted up (Fig. 2d) & $p_{t}(\phi) e^{-3 / 7}$ & 20 & $1-0.4 e^{-(\phi / 40)^{2}}$ \\
WS & whole stratosphere, uniform forcing & $p_{t}(\phi)$ & -1000 & 1 \\
\hline
\end{tabular}

vertical and latitudinal structure of the thermal forcing, and their values are given in Table A1 for each set of integrations. Note, the bracketed term in (A8) is approximately one for $p_{2}<p<p_{1}$ and zero elsewhere.

There is no topography in this model. For $\sigma>\sigma_{b}$, winds are linearly damped as in Held and Suarez (1994) but with a surface time scale of 0.5 days. We apply a sponge layer top and $\nabla^{6}$ hyperviscosity identical to that in Polvani and Kushner (2002).

\section{REFERENCES}

Bohren, C. F., and B. A. Albrecht, 1998: Atmospheric Thermodynamics. Oxford University Press, $402 \mathrm{pp}$.

Butler, A. H., D. W. J. Thompson, and R. Heikes, 2010: The steadystate atmospheric circulation response to climate change-like thermal forcings in a simple general circulation model. $J$. Climate, 23, 3474-3496.

Forster, P. M. D., and K. P. Shine, 2002: Assessing the climate impact of trends in stratospheric water vapor. Geophys. Res. Lett., 29, 1086, doi:10.1029/2001GL013909.

Fueglistaler, S., A. E. Dessler, T. J. Dunkerton, I. Folkins, Q. Fu, and P. W. Mote, 2009: Tropical tropopause layer. Rev. Geophys., 47, RG1004, doi:10.1029/2008RG000267.

Haigh, J. D., M. Blackburn, and R. Day, 2005: The response of tropospheric circulation to perturbations in lower-stratospheric temperature. J. Climate, 18, 3672-3685.

Held, I. M., and M. J. Suarez, 1994: A proposal for the intercomparison of the dynamical cores of atmospheric general circulation models. Bull. Amer. Meteor. Soc., 75, 1825-1830.

Hoskins, B. J., and A. J. Simmons, 1975: A multi-layer spectral model and the semi-implicit method. Quart. J. Roy. Meteor. Soc., 101, 637-655, doi:10.1002/qj.49710142918.

Hurst, D. F., S. J. Oltmans, H. Vömel, K. H. Rosenlof, S. M. Davis, E. A. Ray, E. G. Hall, and A. F. Jordan, 2011: Stratospheric water vapor trends over Boulder, Colorado: Analysis of the 30 year Boulder record. J. Geophys. Res., 116, D02306, doi:10.1029/2010JD015065.

Johanson, C. M., and Q. Fu, 2009: Hadley cell widening: Model simulations versus observations. J. Climate, 22, 2713-2725.

Joshi, M. M., A. J. Charlton, and A. A. Scaife, 2006: On the influence of stratospheric water vapor changes on the tropospheric circulation. Geophys. Res. Lett., 33, L09806, doi:10.1029/2006GL025983.

Lorenz, D. J., and E. T. DeWeaver, 2007: Tropopause height and zonal wind response to global warming in the IPCC scenario integrations. J. Geophys. Res., 112, D10119, doi:10.1029/2006JD008087.
Maycock, A. C., K. P. Shine, and M. M. Joshi, 2011: The temperature response to stratospheric water vapour changes. Quart. J. Roy. Meteor. Soc., 137, 1070-1082, doi:10.1002/qj.822.

Oinas, V., A. A. Lacis, D. Rind, D. T. Shindell, and J. E. Hansen, 2001: Radiative cooling by stratospheric water vapor: Big differences in GCM results. Geophys. Res. Lett., 28, 27912794, doi:10.1029/2001GL013137.

Peixoto, J. P., and A. H. Oort, 1992: Physics of Climate. American Institute of Physics, $520 \mathrm{pp}$.

Polvani, L. M., and P. J. Kushner, 2002: Tropospheric response to stratospheric perturbations in a relatively simple general circulation model. Geophys. Res. Lett., 29, 1114, doi:10.1029/ 2001 GL014284.

Randel, W. J., F. Wu, H. Vömel, G. E. Nedoluha, and P. M. D. Forster, 2006: Decreases in stratospheric water vapor after 2001: Links to changes in the tropical tropopause and the Brewer-Dobson circulation. J. Geophys. Res., 111, D12312, doi:10.1029/2005JD006744.

Rosenlof, K. H., and Coauthors, 2001: Stratospheric water vapor increases over the past half-century. Geophys. Res. Lett., 28 , 1195-1198, doi:10.1029/2000GL012502.

Schneider, T., 2004: The tropopause and the thermal stratification in the extratropics of a dry atmosphere. J. Atmos. Sci., 61, 1317-1340.

_ , and C. C. Walker, 2006: Self-organization of atmospheric macroturbulence into critical states of weak nonlinear eddyeddy interactions. J. Atmos. Sci., 63, 1569-1586.

Seidel, D. J., Q. Fu, W. J. Randel, and T. J. Reichler, 2008: Widening of the tropical belt in a changing climate. Nat. Geosci., 1, 21-24, doi:10.1038/ngeo.2007.38.

Simmons, A. J., and D. M. Burridge, 1981: An energy and angularmomentum conserving vertical finite-difference scheme and hybrid vertical coordinates. Mon. Wea. Rev., 109, 758-766.

Smith, C. A., J. D. Haigh, and R. Toumi, 2001: Radiative forcing due to trends in stratospheric water vapour. Geophys. Res. Lett., 28, 179-182, doi:10.1029/2000GL011846.

Solomon, S., D. Qin, M. Manning, M. Marquis, K. Averyt, M. M. B. Tignor, H. L. J. Miller, and Z. Chen, Eds., 2007: Climate Change 2007: The Physical Science Basis. Cambridge University Press, $1008 \mathrm{pp}$

, K. H. Rosenlof, R. W. Portmann, J. S. Daniel, S. M. Davis, T. J. Sanford, and G. K. Plattner, 2010: Contributions of stratospheric water vapor to decadal changes in the rate of global warming. Science, 327, 1219-1223, doi:10.1126/science. 1182488.

Williams, G. P., 2006: Circulation sensitivity to tropopause height. J. Atmos. Sci., 63, 1954-1961.

World Meteorological Organization, 1957: Meteorology-A threedimensional science. WMO Bull., 4, 134-138. 